\title{
Proteomic Profiling
}

National Cancer Institute

\section{Source}

National Cancer Institute. Proteomic Profiling. NCI Thesaurus. Code C62669.

A laboratory procedure that determines both the identity and levels of expression of the proteins in a biological sample or specimen. 